\title{
Synthesis of non-linear controller to energy efficiency for damped-elastic-jointed inverted pendulum
}

\author{
C.X. Nguyen ${ }^{1}{ }^{*}$ Th.Tr. Le $e^{4}$, Ph.C. Tran ${ }^{1}$, A.D. Lukianov ${ }^{2}$, Kh.D. Truong ${ }^{1}$ and T.D. Pham ${ }^{3}$ \\ ${ }^{1}$ Department of Automation and Computing Techniques, Le Quy Don Technical University, Vietnam \\ ${ }^{2}$ Department of Automation of production processes, Don State Technical University, \\ Rostov-on-Don, Russia \\ ${ }^{3}$ Department of Technology, equipment and aerospace, Le Quy Don Technical University, Vietnam \\ ${ }^{4}$ Controls, Automation in Production and Improvement of Technology Institute, 89B Ly Nam De Street, \\ Hanoi city, Vietnam
}

\begin{abstract}
In the paper we propose a synthetic way of the nonlinear controller for the damped-elastic-jointed inverted pendulum. The controller is designed based on the synergistic approach, the global variables are built on the asymptotically stable manifolds and the fast acting objective function is energy efficient. The effectiveness of the export method is proven through simulation results and compared with other methods.
\end{abstract}

\section{Introduction}

Flexible mechanical systems have been attracting the attention of the research community due to their light weight, smaller energy consumption and reduced fabrication costs compared to traditional hard structures. In addition, the flexible mechanisms are particularly suitable for human-robot interactions and operate in unstructured environments due to their intrinsic compliance. Typically, flexibility can be limited to adjoining non-coupling hard couplings or can be achieved by using compliant links. In a nutshell, flexible mechanisms are often undervalued because they possess more DOF than actuators. In this paper, damped-elasticjointed inverted pendulum are considered, which have been used as a model for wheeled robots [1], robotic fingers and the operator. [2], and the structures are foldable [3]. All system application objects are energy constrained ones. Therefore, the control system requirements for these objects both ensure the consistent quality of the control system and also ensure the energy efficiency.

The damped-elastic-jointed inverted pendulum is the model used in many studies. This feature of the system is an unstable nonlinear system commonly used to study and test the implementation of control algorithms [4-7]. Many studies to build control rules have been successfully designed for inverted pendulum systems, such as the PID for a one-degree inverted pendulum [7]. In the studies [9], the authors used the fuzzy control law to stabilize the

\footnotetext{
* Corresponding author: nguyenxuanchiem83@gmail.com
} 
pendulum in the vertical position when the effect of external force and the fuzzy controller is optimized by the incremental algebra for the control results. good controls. More recently, a more intuitive structure maintenance design similar to the PID was proposed in [3-8] and applied to a flexible inverted pendulum in [5]. However, the above studies are not interested in control signals, which high mobility applications require.

In this paper, the author presents the nonlinear controller design method based on the fusion approach given by scientist Kolecnikov A.A. $[13,15]$. In the measurement of the target function selected by the authors group, it has fast impact and controls energy efficiency. The effect of the designed control law is shown through the simulation results when changing the parameters. At the same time, the authors also compared the proposed control quality with other controllers to show that in addition to the improved control quality, the cost of the control signal is reduced. The rest of the paper is organized as follows: Part 2 presents the mathematical model of the damped-elastic-jointed inverted pendulum stitches and draws the linear model. Part 3 details design techniques of control law based on fusion approach, Part 4 presents simulation results and discusses implemented control law. Finally, Part 5 presents the conclusion of this article.

\section{The damped-elastic-jointed inverted pendulum}

The diagram of the system is shown in Figure 1. An inverted pendulum of length $l$ has a concentrated mass $m$ at its end. A spring (of constant stiffness $k$ ) and a damper (of constant damping factor $c$ ) connect the inverted pendulum to a fixed base to avoid a large rotation angle $\varphi$. A periodic follower force $(F=P 1+P 2 \cos \omega t)$ acts on the mass $\mathrm{m}$ and makes an angle $\gamma \varphi$ with the vertical axis. A control moment $u$, applied at the base of the inverted pendulum, brings the angle $\varphi$ to zero position.

The kinetic energy $T$, the potential energy $H$ and the dissipative function $\Phi$ of the system can be expressed in the following forms $[9,10]$ :

$$
\begin{gathered}
T=\frac{1}{2} m\left(\dot{x}_{m}^{2}+\dot{y}_{m}^{2}\right)=\frac{1}{2} m l^{2} \dot{\varphi}^{2} \\
H=m g y_{m}+\frac{1}{2} k \varphi^{2}=m g l \cos (\varphi) \frac{1}{2} k \varphi^{2} \\
\Phi=\frac{1}{2} c \dot{\varphi}^{2}
\end{gathered}
$$

where $x_{m}=l \sin \varphi, \mathrm{y}_{\mathrm{m}}=1 \cos \varphi$ and $\mathrm{g}$ is the gravity acceleration.

Using the Lagrange equations of the second type:

$$
\frac{d}{d t}\left(\frac{\partial T}{\partial \dot{\varphi}}\right)-\frac{\partial T}{\partial \varphi}=-\frac{\partial H}{\partial \varphi}-\frac{\partial \Phi}{\partial \varphi}+M
$$

The differential equation of motion for this system can then be written in the form:

$$
m l^{2} \ddot{\varphi}+c \dot{\varphi}+k \varphi-m g l \sin \varphi=u+F l \sin (\varphi-\gamma \varphi)
$$

Denoting $\left[x_{1}, x_{2}\right]^{\mathrm{T}}=[\varphi, \dot{\varphi}]^{\mathrm{T}}$, Equation (5) can be rewritten into state-space form as 


$$
\left\{\begin{array}{l}
\dot{x}_{1}=x_{2} \\
\dot{x}_{2}=-\frac{k}{m l^{2}} x_{1}+\frac{g}{l} \sin x_{1}+\frac{F \sin \left(x_{1}-\gamma x_{1}\right)}{m l}-\frac{c}{m l^{2}} x_{2}+\frac{u}{m l^{2}}
\end{array}\right.
$$

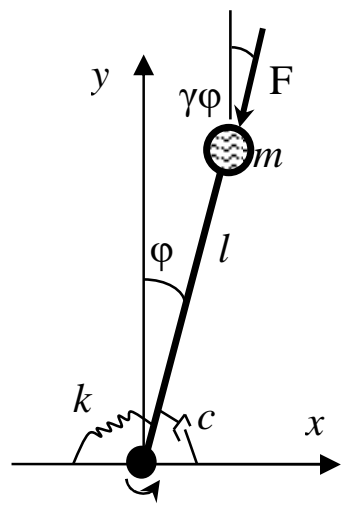

$\mathrm{u}$

Fig.1. Configuration of the inverted pendulum system.

System model parameters are presented in the study [9]: $\mathrm{m}=2 \mathrm{~kg}, \mathrm{k}=2 \mathrm{Nm}, 1=1.5 \mathrm{~m}$, $\mathrm{P} 1=10 \mathrm{~N}, \mathrm{P} 2=1 \mathrm{~N}, \mathrm{c}=0.005 \mathrm{Nms}, \omega=\pi / 2$ and $\mathrm{g}=9.81 \mathrm{~m} / \mathrm{s} 2$. Construct the system phase plane with initial state $\mathrm{x} 1(0)=0.02, \mathrm{x} 2(0)=0.0$ and when the parameter $\gamma=\{0.1,0.5,0.9\}$ get the system phase plane portrait in turn. figures 2 (a), 2 (b), 2 (c). From the phase portrait of the system when $\gamma$ changes there is a turbulent, unstable motion. So a nonlinear control rule will result in a better response quality of the system.

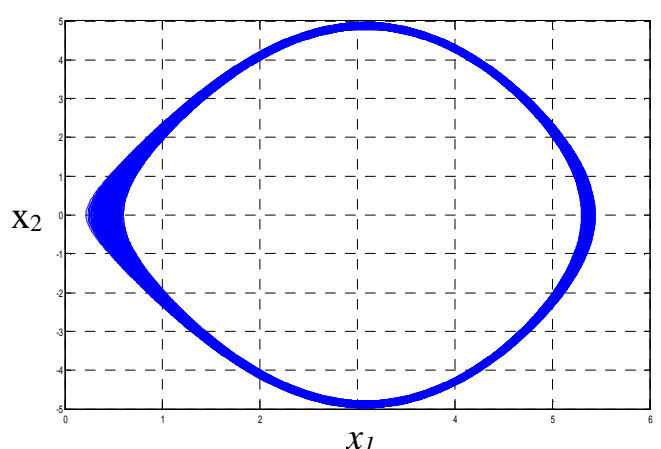

a)

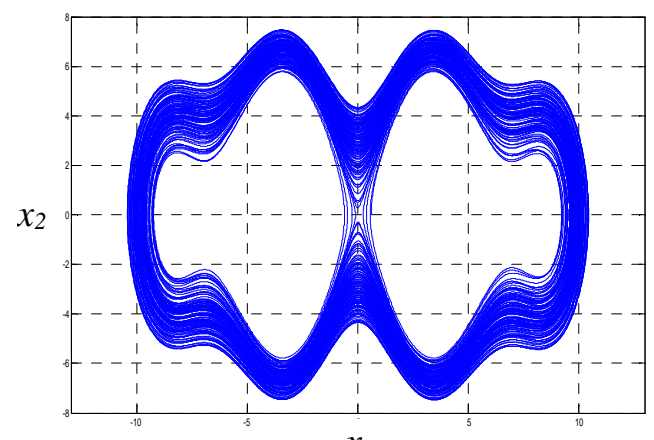

$\underset{\mathrm{b})}{x_{1}}$ 


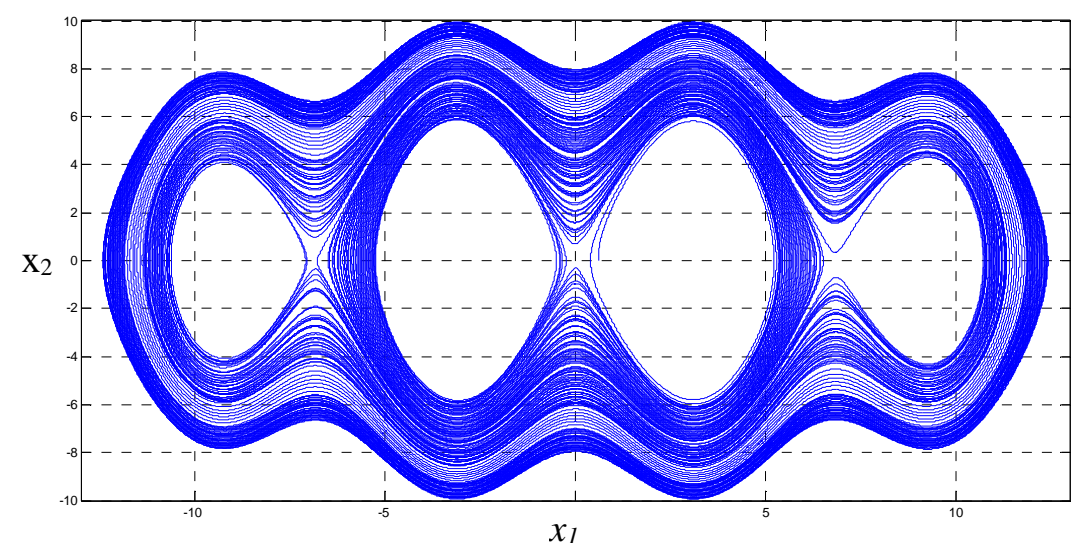

c)

Fig. 2. Portrait of the phase plane of the inverted pendulum system when $\gamma$ receives different values: a) $\gamma=0.9$; b) $\gamma=0.5$; a) $\gamma=0.1$;

\section{Synthesis of energy efficient angular controller based on synergistic method ADAR}

\subsection{Approach to quasi-time-optimal control $[12,13]$}

By using this approach, the problem of synthesizing control law for a wide spectrum of nonlinear objects can be solved. It is reported that the synthesized control law has several advantages, such as: quasi-optimal minimum-time, asymptotic stability and robustness.

The concept of rapid-impact nearly-optimal control is shown below. Suppose model system state form taken by Jordan in the form:

$$
\left\{\begin{array}{l}
\dot{x}_{i}=f_{i}\left(x_{1}, x_{2}, \ldots x_{i+1}\right), i=\overline{1, n-1} \\
\dot{x}_{n}=f_{n}\left(x_{1}, x_{2}, \ldots x_{n}\right)+u
\end{array}\right.
$$

where $f_{i}(\circ)$ is an analytic function, which means that the derivative exists according to all variables $x_{1}, x_{2}, \ldots x_{i+1}, \forall i<n \rightarrow \partial f_{i} / \partial x_{i+1} \neq 0$, and $u(t)$ is the control signal.

The synthetic methods of rapid-impact nearly-optimal control laws to system (7) is using the differential transformations put the system (7) on the virtual system of equations rank $\mathrm{k}$ has the following form (8):

$$
\left\{\begin{array}{l}
\dot{y}_{1}=-v_{1}^{m} h\left(y_{1}, \varepsilon_{1}\right)+y_{2} ; \ldots ; \dot{y}_{k}=v_{k}^{m} h\left(y_{k}, \varepsilon_{k}\right)+y_{k+1} \\
\dot{y}_{k+1}=-\frac{y_{k+1}}{\varepsilon_{k+1}}+y_{k+2} ; \ldots ; \dot{y}_{n-1}=-\frac{y_{n-1}}{\varepsilon_{n-1}}+y_{n} \\
\dot{y}_{n}=-\frac{y_{n}}{\varepsilon_{n}}
\end{array}\right.
$$

From this equation, proceed to solve the equation with $y_{1}=\phi\left(x_{1}\right)$ we get the rapid-impact nearly-optimal control laws of the desired variable. 


\subsection{Synergistic approach to the analytical design of aggregated regulators}

The analytical design of aggregated controllers (ADAR) proposed in $[14,15]$ in the sense of ensuring asymptotic stability and given dynamic properties of transients is based on the introduction of functionals of the form (9):

$$
J=\int_{0}^{\infty} F(\psi, \dot{\psi}) d t
$$

where $F(\psi, \dot{\psi})$ - continuously differentiable function with all arguments; $\psi\left(x_{1}, \ldots x_{\mathrm{n}}\right)$ aggregated macro-variable, representing some arbitrary or piecewise of differentiable continuous function of phase coordinates $\left(x_{1}, \ldots, x_{n}\right)$, moreover $\psi(0, \ldots, 0)=0$.

The essence of the synergetic approach is to construct an accompanying function in such a way as to determine in the phase space of the non-linear object under consideration a certain set of points to which all close motion paths are attracted. Such a set of points is usually called an attractor [11]. The above actually corresponds to the basic principle of synergetics - the principle of submission, on which the theory of self-organization of nonlinear dynamic systems is built $[14,15]$.

In particular, for a non-linear SISO object, with scalar control, it is advisable to choose the following quadratic form as an integrand in (9):

$$
F(\psi, \dot{\psi})=m^{2} \varphi^{2}(\psi)+c^{2} \dot{\psi}^{2}(t)
$$

With the function $\varphi(\psi)$ must satisfy the following conditions:

1. Single-valuedness, continuity and differentiability for all values ;

2. $\varphi(0)=0$;

3. $\varphi(\psi) \psi>0$ at any $\varphi \neq 0$

Selection of immutable $\psi$ manifolds of the form:

$$
\psi=x_{n}+\phi\left(x_{1}, \ldots, x_{n-1}\right)=0
$$

The manifold (11) must satisfy the solutions of the functional equation:

$$
c \dot{\psi}(t)+m \varphi(\psi(t))=0
$$

Solving this equation we get the control law for the system.

\subsection{Synthesis of angle stability control laws for The damped-elastic-jointed inverted pendulum hat are energy efficient.}

The inverted pendulum non-liner control system is based on the closed-loop system shown in Figure 3.

To control the system, we assume the universe of discourse for the two state variables to be $-1 \mathrm{rad}<x_{1}<1 \mathrm{rad}$ and $-2 \mathrm{rad} / \mathrm{s}<x_{2}<2 \mathrm{rad} / \mathrm{s}$. The universe of discourse for the control moment is $-50 \mathrm{Nm}<u<50 \mathrm{Nm}$ [9].

Saving energy in control systems with limited power supply is a current issue that has received much attention from researchers. To synthesize fast acting control laws and reduce energy consumption is a difficult requirement. Because fast acting requires a great deal of energy consumption and when reducing energy consumption, fast acting is not a priority. With the aim of both ensuring fast impact and minimizing energy consumption, the authors propose a method to synthesize control laws based on an integrated approach. 


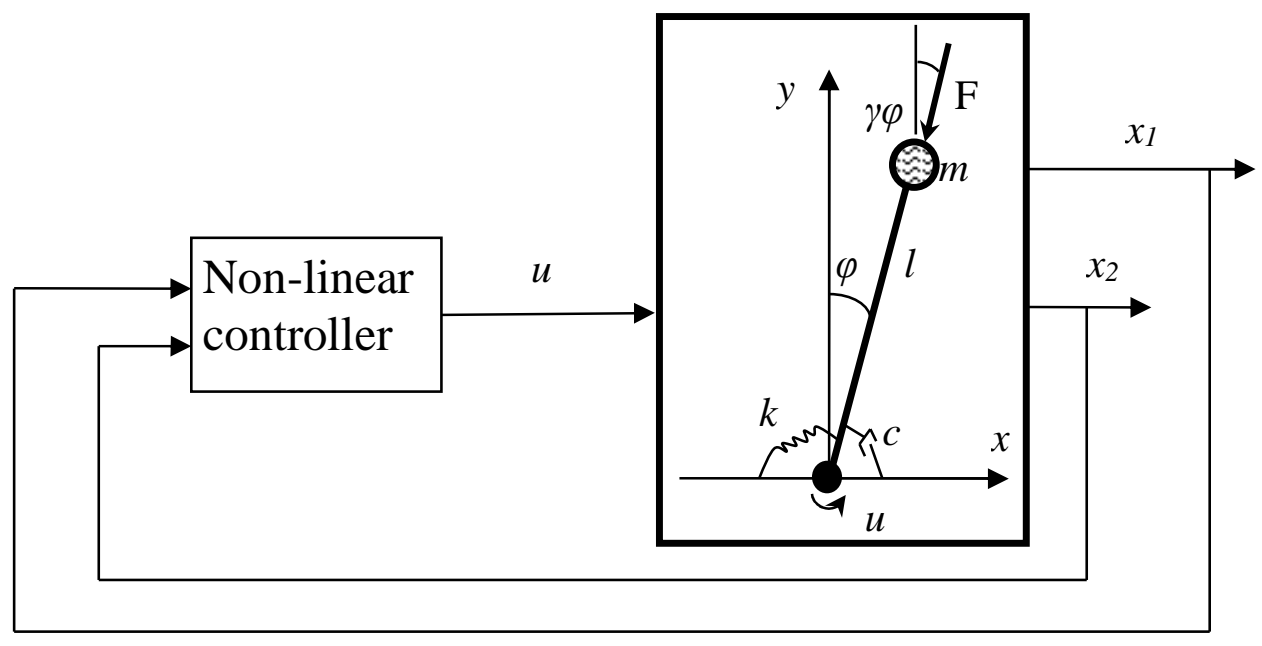

Fig. 3. System control structure diagram

Select macro-variables with $\psi=T_{1} x_{2}+x_{1}$. Select the target function for the equations (6) of the form (9):

$$
J=\int_{0}^{\infty}\left(\varphi^{2}(\psi)+T_{2}^{2} \dot{\psi}^{2}(t)\right) d t
$$

Where

$$
\varphi(\psi)=\frac{\psi}{\sqrt{\psi^{2}+\varepsilon_{2}^{2}}}, \dot{\psi}=-\frac{T_{1} k}{m l^{2}} x_{1}+\frac{T_{1} g}{l} \sin x_{1}+\frac{T_{1} F \sin \left(x_{1}-\gamma x_{1}\right)}{m l}-\frac{T_{1} c}{m l^{2}} x_{2}+\frac{T_{1} u}{m l^{2}}+x_{2} ;
$$

From the target function as chosen when away from the equilibrium point $\varphi^{2}(\psi) \rightarrow 1$, the target function becomes $\quad J=\int_{0}^{\infty}\left(1+T^{2} \dot{\psi}^{2}(t)\right) d t$ and in the target function that contains the signal component that drives $\mathrm{u}$. Therefore, the control target is also meant to ensure that the control signal is minimized.

The law of synthetic control must satisfy the functional equation (14) $[14,15]$ :

$$
\varphi(\psi)+T_{2} \dot{\psi}(t)=0
$$
[15]:

Solving equation (14) and receiving the control law in analytic form has the following form

$$
u=k x_{1}-m l g \sin x_{1}-l F \sin \left(x_{1}-\gamma x_{2}\right)+\left(c-\frac{m l^{2}}{T_{2} T_{1}}\right) x_{2}-\frac{m l^{2}}{T_{2} T_{1}} \frac{T_{1} x_{2}+x_{1}}{\sqrt{\left(T_{1} x_{2}+x_{1}\right)^{2}+\varepsilon^{2}}}
$$

\section{Simulating results}

The parameters of mathematical model (6) is presented in Part 1. The parameters of the suboptimal controller are: $v=1.5, \varepsilon 1=1, \varepsilon 2=0.02$. The proposed controller parameters include: $T 1=1.5, T 2=1.5, \varepsilon=0.1$; The simulation was performed on Simulink-Matlab software with the initial condition of the angle of The damped-elastic-jointed inverted pendulum $x 1=0.6(\mathrm{rad}), x 2=0(\mathrm{rad} / \mathrm{s})$ with the purpose of bringing the system to the balance position $(x 1=0, x 2=0)$ with the quasi-optimality and energy-saving. The simulation results were performed with two controllers shown in fig. 4: Quasi-time optimal controller (KOB) and proposed controller (Syner). The controller is selected so that the system's angular response parameters are approximately the same. Figure fig. 4 (a) is the system status response, and Fig. 
4 (b) is the system control signal. From the results we see that the angular responses of the two controllers are quite similar, but the velocity responses are different, the overshoot of the KOB controller is greater than the proposed controller (Syner), although the deceleration rate The speed is fast, but the response time is equal. The control signal of the KOB controller has a large initial value $(u=-45.19 \mathrm{Nm})$, while the proposed controller gives a maximum value of the control signal $(u=28.6 \mathrm{Nm})$. Control energy for control law $W_{K O B}=6.38(\mathrm{~J}), W_{\text {Syne }}=6.12(\mathrm{~J})$. From here it shows that with the same control quality of the typical state, which is the deflection angle, the control signal of the proposed method gives better efficiency of energy.

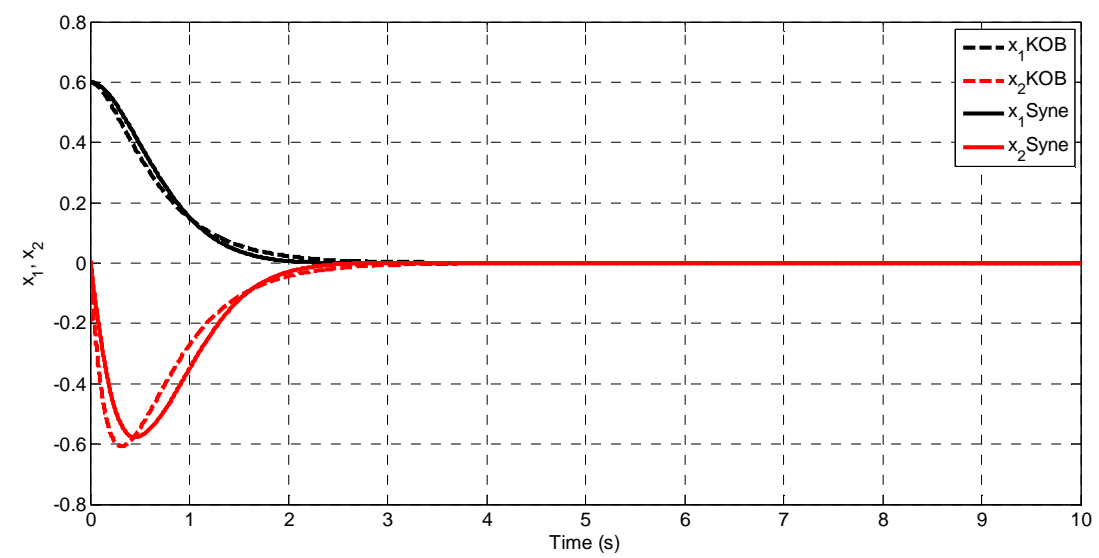

Fig. 4. Response to system state variables

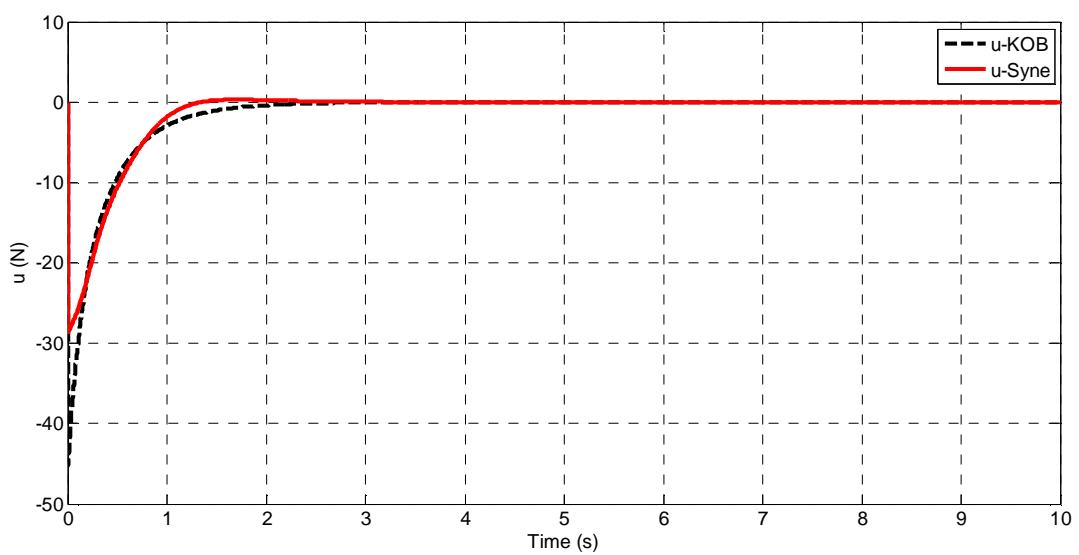

Fig. 5. Response to control signals

\section{Conclusion}

The controller design method based on a fusion approach with a functional equation with fast acting and energy efficiency has improved control quality and reduced control energy consumption. Comparison with KOB law shows the efficiency of the proposed method when the same quality of state response but the initial control signal is smaller than $16.59(\mathrm{~N})$ and the energy is less than $0.26(\mathrm{~J})$. In addition, the choice of manifolds to ensure the system is optimized for energy is also a factor to consider, so the next development direction of the 
authors team will study manifolds for efficiency in terms of energy efficiency and controller construction on embedded systems.

\section{References}

1. A.C. Satici, A. Donaire, B. Siciliano, Intrinsic dynamics and total energy-shaping control of the ballbot system, Int. J. Control. (2016) 1-14. doi:10.1080/00207179.2016.1264630.

2. X.-Z. Lai, C.-Z. Pan, M. Wu, S.X. Yang, Unified control of n-link underactuated manipulator with single passive joint: A reduced order approach, Mech. Mach. Theory. 56 (2012) 170-185. doi:10.1016/J.MECHMACHTHEORY.2012.05.011.

3. G. Radaelli, R. Buskermolen, R. Barents, J.L. Herder, Static balancing of an inverted pendulum with prestressed torsion bars, Mech. Mach. Theory. 108 (2017) 14-26. doi:10.1016/J.MECHMACHTHEORY.2016.10.005.

4. Lal Bahadur Prasad, Barjeev Tyagi Hari, Om GuptaOptimal, Control of Nonlinear Inverted Pendulum System Using PID Controller and LQR: Performance Analysis Without and With Disturbance Input. International Journal of Automation and Computing. 11(6), December 2014, 661-670.

5. P.S. Gandhi, P. Borja, R. Ortega, Energy shaping control of an inverted flexible pendulum fixed to a cart, Control Eng. Pract. 56 (2016) 27-36. doi:10.1016/j.conengprac.2016.07.010

6. R. Mehra, S.G. Satpute, F. Kazi, N.M. Singh, Control of a class of underactuated mechanical systems obviating matching conditions, Automatica. 86 (2017) 98-103. doi:10.1016/J.AUTOMATICA.2017.07.033.A. Donaire, R. Mehra, R. Ortega, S.

7. P. Borja, R. Cisneros, R. Ortega, A constructive procedure for energy shaping of portHamiltonian systems, Automatica. $72 \quad$ (2016) 230-234. doi:10.1016/J.AUTOMATICA.2016.05.028.

8. Satpute, J.G. Romero, F. Kazi, N.M. Singh, Shaping the Energy of Mechanical Systems Without Solving Partial Differential Equations, IEEE Trans. Automat. Contr. 61 (2016) 1051-1056. doi:10.1109/TAC.2015.2458091.

9. Hai-Le Bui, Duc-Trung Tran, Nhu-Lan Vu. Optimal fuzzy control of an invertedpendulum. Journal of Vibration and Control 18(14) 2097-2110. DOI: 10.1177/1077546311429053

10. Galan J, Fraser WB, Acheson DJ and Champneys AR (2005) The parametrically excited upside-down rod: an elastic jointed pendulum model. Journal of Sound and Vibration 280: 359-377.

11. C X Nguyen, A D Lukianov, T D Pham and A D Nguyen. Synthesis of a nonlinear control law with efficiency energy for the self-balancing two wheeled vehicle. IOP Conf. Series: Materials Science and Engineering $900 \quad$ (2020) 012002 doi:10.1088/1757899X/900/1/012002.

12. Nguyen X. C.,Phan H.N.. Design controler of the quasi time optimization approach forstabilizing and trajectory tracking of inverted pendulum. MATEC Web Conf.Volume 226, 2018 XIV International ScientiÒc-Technical Conference "Dynamic of Technical Systems" (DTS-2018).

13. Chiem Nguyen Xuan, Hai Phan Nguyen, Long Hoang Duc, Khoa Truong Dang, Le Minh Kien, Thuy Xuan Pham. Building quasi-time-optimal control laws for ball and beam system. 2019 3rd International Conference on Recent Advances in Signal Processing, Telecommunications \&Computing (SigTelCom).

14. Kolesnikov A.A. Synergetic control theory - Taganrog: TRTU, Energoatomizdat, 1994 $344 \mathrm{p}$.

15. Kolesnikov A.A. Synergetics and problems of control theory: collection of articles. scientific. tr. Ed. A.A. Kolesnikov. M .: FIZMATLIT, (2004) 